\title{
Simulations and Experiments Study on the Mechanical Quality Factor of the Cylindrical Shell Fused Silica Resonators
}

\author{
Pengbo Xiao, Yao Pan, Tianliang Qu*, Yun Huang \\ Department of Opto-electronic Science and Engineering, National University of Defense \\ Technology, Changsha 410073, China
}

Keywords: cylindrical shell fused silica resonators; damping loss; quality factor

\begin{abstract}
The cylindrical shell fused silica resonator is the critical component of a cylindrical vibratory gyroscope, and the quality factor is a key parameter to evaluate the performance of the cylindrical resonator. This paper focuses on the quality factor of the cylindrical shell fused silica resonator. The influence of air damping, surface defect loss, thermoelastic dissipation and internal friction loss on the quality factor are studied through simulations, which provide a theoretical trend for quality factor experiments. Quality factor is further explored by means of changing different damping loss of the resonator, for example, annealing, chemical polishing or vacuum pumping in experiments and the $\mathrm{Q}$ factor of the experimental cylindrical shell fused silica resonator has been optimized comprehensively, about 110 times.
\end{abstract}

\section{Introduction}

With the advantages of high precision, small size, light weight, long service life and low power consumption, the Coriolis vibratory gyroscope $(\mathrm{CVG})$ is becoming widely used [1][2][3]. As an important kind of CVG, cylindrical vibratory gyroscope is being intensively studied since its comprehensive advantages in precision, cost and size [4][5][6]. Resonator is the critical component of a CVG, and the quality factor ( $Q$ factor) is a key parameter to evaluate the performance of the cylindrical resonator which links closely with the precision and sensitivity of CVG.

For cylindrical shell resonator, the $\mathrm{Q}$ factor equals to the ratio of the total energy stored in the resonator to the energy loss of the resonator in a cycle [7], that is

$$
\mathrm{Q}=\frac{2 \pi E_{\text {total }}}{\Delta E}=\frac{2 \pi E_{\text {total }}}{\sum_{i=1}^{n} \Delta E_{\mathrm{i}}}
$$

where $E_{\text {total }}$ and $\Delta E$ are respectively the total energy and the energy loss per resonant cycle. Since different energy loss affecting the $\mathrm{Q}$ factor acts as the denominator, 1/Q can be used to represent the energy loss.

$$
\frac{1}{\mathrm{Q}}=\sum_{\mathrm{i}=1}^{\mathrm{n}} \frac{1}{\mathrm{Q}_{\mathrm{i}}}
$$

$1 / \mathrm{Q}_{\mathrm{i}}$ represents the effect of different loss on $\mathrm{Q}$ factor. For cylindrical shell resonators, energy loss includes air damping, $1 / \mathrm{Q}_{\text {air }}$, surface defect loss, $1 / \mathrm{Q}_{\mathrm{sur}}$, thermoelastic dissipation, $1 / \mathrm{Q}_{\mathrm{TED}}$, internal friction loss, $1 / \mathrm{Q}_{\text {fri }}$ and anchor loss, $1 / \mathrm{Q}_{\text {anchor }}[8]$.

$$
\frac{1}{\mathrm{Q}}=\frac{1}{\mathrm{Q}_{\text {air }}}+\frac{1}{\mathrm{Q}_{\text {sur }}}+\frac{1}{\mathrm{Q}_{\mathrm{TED}}}+\frac{1}{\mathrm{Q}_{\text {fri }}}+\frac{1}{\mathrm{Q}_{\text {anchor }}}
$$

Using Matlab and Mathematica, the influence of air damping, surface defect loss, thermoelastic dissipation and internal friction loss on $\mathrm{Q}$ factor are studied through simulations. The processing size of resonators are also determined according to the simulation results. $\mathrm{Q}$ factor is further explored in experiments by means of changing different damping loss of the resonator. In this paper, air damping is decreased by vacuum pumping, surface defect loss is decreased by chemical polishing, thermoelastic dissipation and internal friction loss are both decreased by annealing. Most resonators are made in metal, but high damping of metal limits $Q$ factors, so we propose a monolithic cylindrical 
resonator made by high purity fused silica [9]. This material is highly isotropic and has low internal friction, therefore it is preferred in high-Q resonators. Finally, the $\mathrm{Q}$ factor of the experimental resonator has been optimized comprehensively, about 110 times.

\section{Resonator model}

The vibratory type of cylindrical shell resonator shows like the cantilever mode, so it can be approximately represented by the beam model. In this chapter, a new resonator model is proposed, containing the bottom vibration of the resonator based on the beam model. The definition of coordinate system is showed in Fig. 1 and the new resonator model is showed in Fig.2. Each parameter meaning can be found in table 1 .

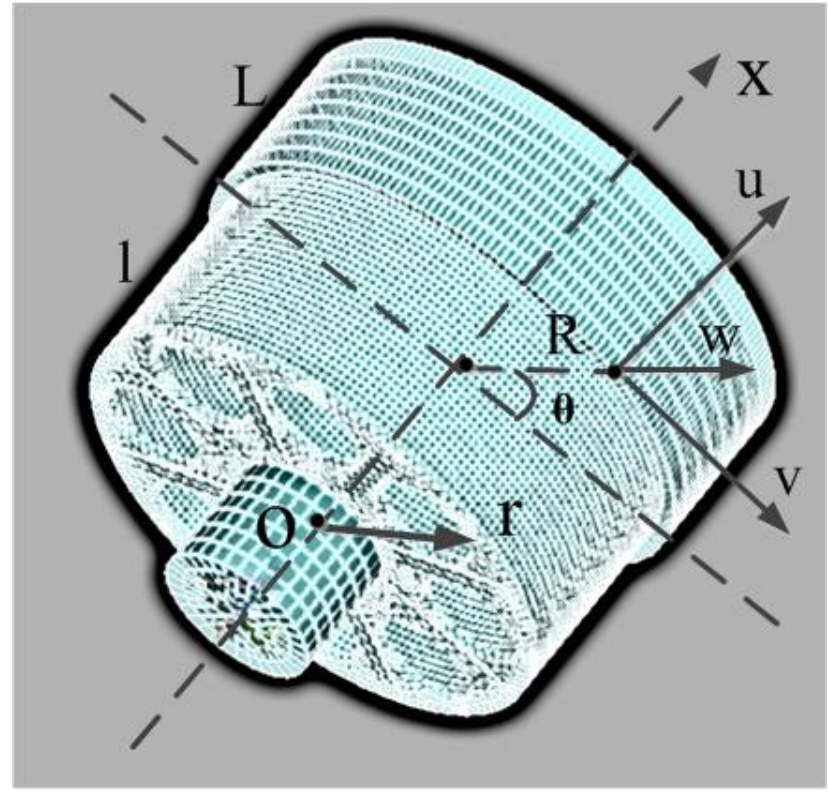

Fig. 1 Coordinate system of the resonator model

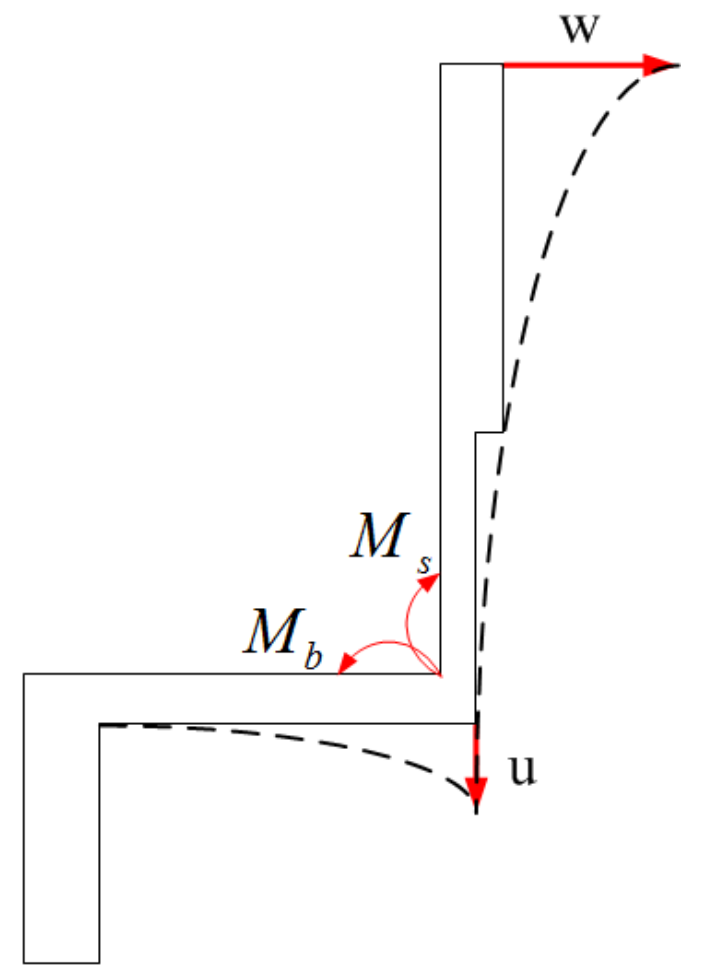

Fig. 2 The new resonator model

Each parameter meaning can be found in table 1 . 
Table. 1 Each parameter meaning

\begin{tabular}{|c|c|}
\hline Parameter & Meaning \\
\hline$x$ & Axial coordinates \\
\hline$\theta$ & Circumferential angle \\
\hline$r$ & Axial displacement of the resonant ring \\
\hline$u$ & Tangential displacement of the resonant ring \\
\hline$v$ & Radial displacement of the resonant ring \\
\hline$w$ & Time \\
\hline$t$ & Outer radius of the resonant ring \\
\hline$R$ & Height of the resonant ring \\
\hline$L$ & Height of the support structure \\
\hline$l$ & Thickness of the resonant ring \\
\hline$h r$ & Thickness of the support structure \\
\hline$h s$ & Moment produced by support structure \\
\hline$M s$ & Moment produced by bottom \\
\hline$M_{b}$ & \\
\hline
\end{tabular}

The vibration form of the cylindrical shell resonator can be expressed as [10]

$$
\left\{\begin{array}{c}
u=U(x) \cdot \cos (\mathrm{n} \theta) \cdot \cos (\omega t) \\
v=V(x) \cdot \sin (\mathrm{n} \theta) \cdot \cos (\omega t) \\
w=W(x) \cdot \cos (\mathrm{n} \theta) \cdot \cos (\omega t) \\
u_{b}=U_{b}(r) \cdot \cos (\mathrm{n} \theta) \cdot \cos (\omega t)
\end{array}\right.
$$

$u_{b}$ is the radial displacement of bottom, $\mathrm{n}$ is the circumferential wave number and $\omega$ is the vibrational frequency. According to the beam deformation theory, axial vibration of bottom surface is

$$
U_{b}(r)=U_{0} \cdot\left(\frac{r-r_{0}}{R-r_{0}}\right)^{2}
$$

$U_{0}$ is the axial vibration amplitude and $r_{0}$ is the radial coordinate of the bottom polar coordinates.

The axial displacement produced by the lateral wall is very small, so $U(x)$ approximates to $U_{0}$. From cylindrical shell theory, the moment of the side wall and the bottom boundary is equal [11], so

$$
M_{s}(x)=M_{b}(x)
$$

and

$\frac{W_{0}}{U_{0}}=\frac{\left(1-\mu^{2}\right) \cdot D_{b}}{\lambda^{2} \cdot D_{s}} \cdot\left[\frac{2}{\left(R-r_{0}\right)^{2}}+\mu \cdot\left(\frac{2}{R \cdot\left(R-r_{0}\right)}+\frac{\mathrm{n}^{2}}{R^{2}}\right)\right]$

$D_{s}=\frac{\mathrm{E} \cdot h_{s}{ }^{3}}{12\left(1-\mu^{2}\right)}, D_{b}=\frac{\mathrm{E} \cdot h_{b}{ }^{3}}{12\left(1-\mu^{2}\right)}, \lambda=1.875 /(L+l)$, and $\mu, \mathrm{E}$ are Poisson's ratio and Young's modulus respectively.

Therefore, the vibration type function of side wall beam is

$$
W(x)=W_{0}\left\{\cosh (\lambda x)-\cos (\lambda x)-E_{r} \cdot[\sinh (\lambda x)-\sin (\lambda x)]\right\}+\frac{2 x}{R-r_{0}} \cdot U_{0}
$$

Inside, $E_{r}=\frac{\sinh [\lambda(L+l)]-\sin [\lambda(L+l)]}{\cosh [\lambda(L+l)]+\cos [\lambda(L+l)]}$. 


\section{Damping loss simulation}

\subsection{Surface defect loss}

Cylindrical shell resonator is generally processed by grinding, which will inevitably destroy the surface of the shell and bring the surface defect loss. The T.Uchiyama research group classifies the surface defect loss as a kind of thermal elastic loss and gives the calculating formula [12].

$$
\frac{1}{\mathrm{Q}_{\text {sur }}}=2 h_{\text {dam }} \cdot\left(\frac{1}{2 R}+\frac{1}{L+l}\right) \cdot \frac{\mathrm{E} \Gamma \gamma^{2}}{c} \cdot \frac{\omega_{0} \tau}{1+\left(\omega_{0} \tau\right)^{2}}
$$

$c$ is the heat capacity per unit volume, $\gamma$ is the thermal expansion coefficient, $h_{d a m}$ is the thickness of damage layer, $\Gamma$ is the temperature of resonator, $\tau=\psi^{2} \frac{c}{\kappa}, \psi$ is the thermal distribution scale and $\kappa$ is the thermal conductivity.

Using matlab, the surface defect loss is calculated according to formula. Surface defect loss varies with the outer radius of the resonant ring, as showed in Fig.3. Its order of magnitude is about $10^{-5}$ and it decreases with the increase of the outer radius of the resonant ring.

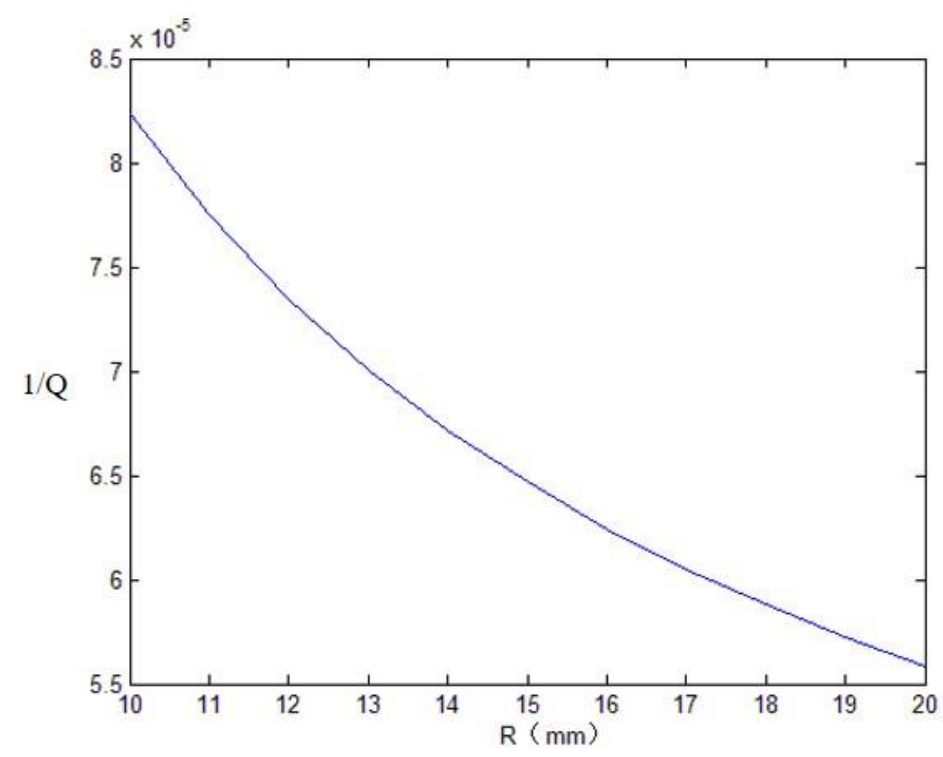

Fig. 3 Surface defect loss varies with the outer radius of the resonant ring

Surface defect loss varies with the ratio of the height of the resonant ring and support structure is showed in Fig. 4. It decreases with the increase of the ratio of the height of the resonant ring and support structure, so when the height of the resonant ring is set a fixed value, the higher the resonant ring, the lower the surface defect loss. 


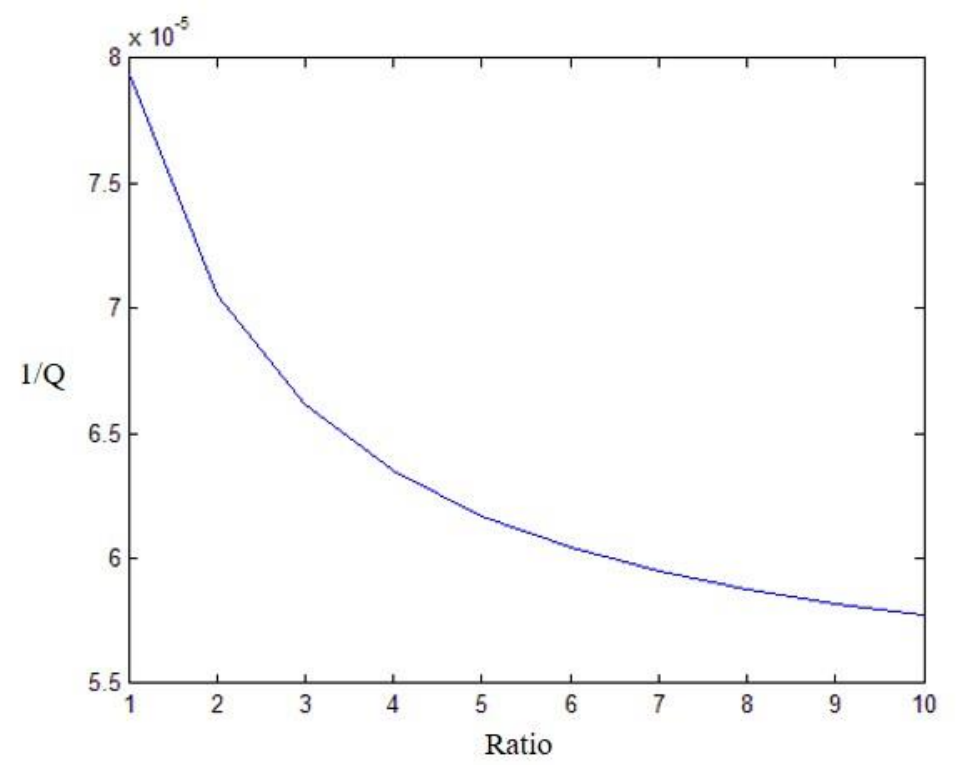

Fig. 4 Surface defect loss varies with the ratio of the height of the resonant ring and support structure 3.2 Thermoelastic dissipation

The cylindrical shell fused silica resonator deforms during vibration, so the material is compressed and stretched to produce different temperature gradients. This internal temperature conduction leads to thermoelastic dissipation and it can be calculated as [13][14][15]

$$
\frac{1}{\mathrm{Q}_{\mathrm{TED}}}=2\left|\frac{\operatorname{Im}\left(\omega_{\mathrm{mn}}\right)}{\operatorname{Re}\left(\omega_{\mathrm{mn}}\right)}\right|
$$

$\omega_{m n}$ is the natural frequency of the resonator, and

$$
\left\{\begin{array}{l}
\operatorname{Re}\left(\omega_{m n}\right)=\frac{1}{R} \sqrt{\frac{\mathrm{E}}{\rho}} \sqrt{\psi} \cos \frac{\Xi}{2} \\
\operatorname{Im}\left(\omega_{m n}\right)=\frac{1}{R} \sqrt{\frac{\mathrm{E}}{\rho}} \sqrt{\psi} \sin \frac{\Xi}{2}
\end{array}\right.
$$

SO

$$
\frac{1}{\mathrm{Q}_{\mathrm{TED}}}=2\left|\frac{\operatorname{Im}\left(\omega_{\mathrm{mn}}\right)}{\operatorname{Re}\left(\omega_{\mathrm{mn}}\right)}\right|=2\left|\tan \frac{\Xi}{2}\right|
$$

Since

$$
\tan \Xi=\frac{\operatorname{Im}\left(\Delta \Omega^{2}{ }_{m n}\right)}{\operatorname{Re}\left(\Delta \Omega^{2}{ }_{m n}\right)+\Omega^{2}{ }_{m n}}
$$

and $\operatorname{Im}\left(\Delta \Omega^{2}{ }_{m n}\right) 、 \operatorname{Re}\left(\Delta \Omega^{2}{ }_{m n}\right) 、 \Omega^{2}{ }_{m n}$ are calculated in the reference, so

$$
\tan \Xi=\frac{2 \tan \frac{\Xi}{2}}{1-\tan ^{2} \frac{\Xi}{2}}=\frac{\operatorname{Im}\left(\Delta \Omega^{2}{ }_{m n}\right)}{\operatorname{Re}\left(\Delta \Omega^{2}{ }_{m n}\right)+\Omega^{2}{ }_{m n}}=a
$$

and

$$
\tan \frac{\Xi}{2}=\frac{\sqrt{a^{2}+1}-1}{a}
$$

SO

$$
\frac{1}{\mathrm{Q}_{\mathrm{TED}}}=2\left|\tan \frac{\Xi}{2}\right|=\frac{2\left(\sqrt{\mathrm{a}^{2}+1}-1\right)}{\mathrm{a}}
$$


Thermoelastic dissipation, as showed in Fig.5, its order of magnitude is about $10^{-3}$ to $10^{-2}$ and it decreases with the increase of the outer radius of the resonant ring.

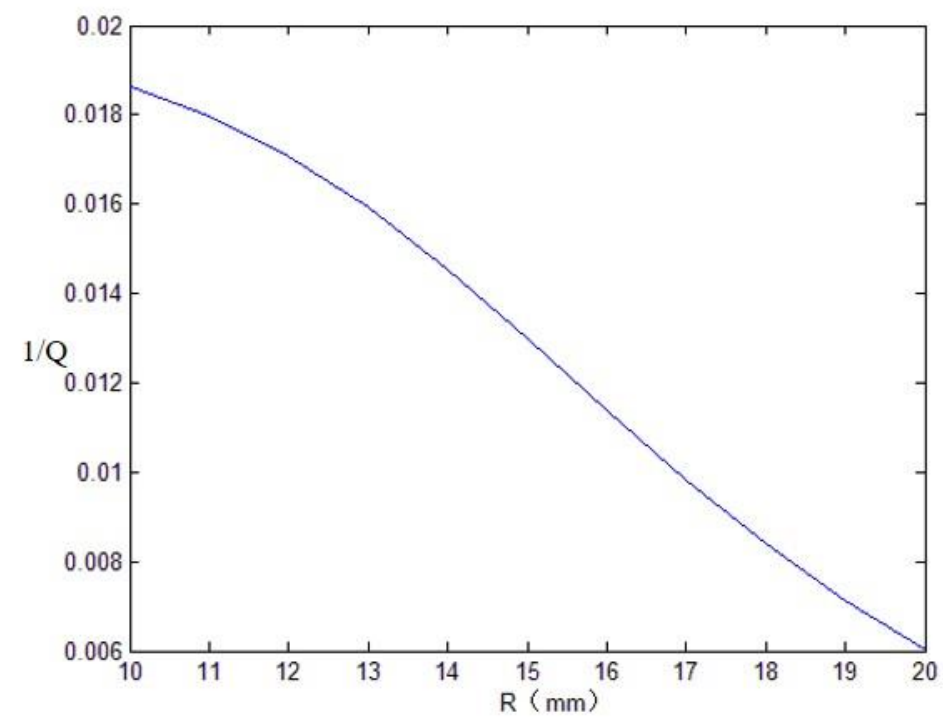

Fig. 5 Thermoelastic dissipation varies with the outer radius of the resonant ring

Thermoelastic dissipation varies with the ratio of the height of the resonant ring and support structure is showed in Fig. 6. It increases with the increase of the ratio of the height of the resonant ring and support structure, so when the height of the resonant ring is set a fixed value, the higher the resonant ring, the higher the thermoelastic dissipation.

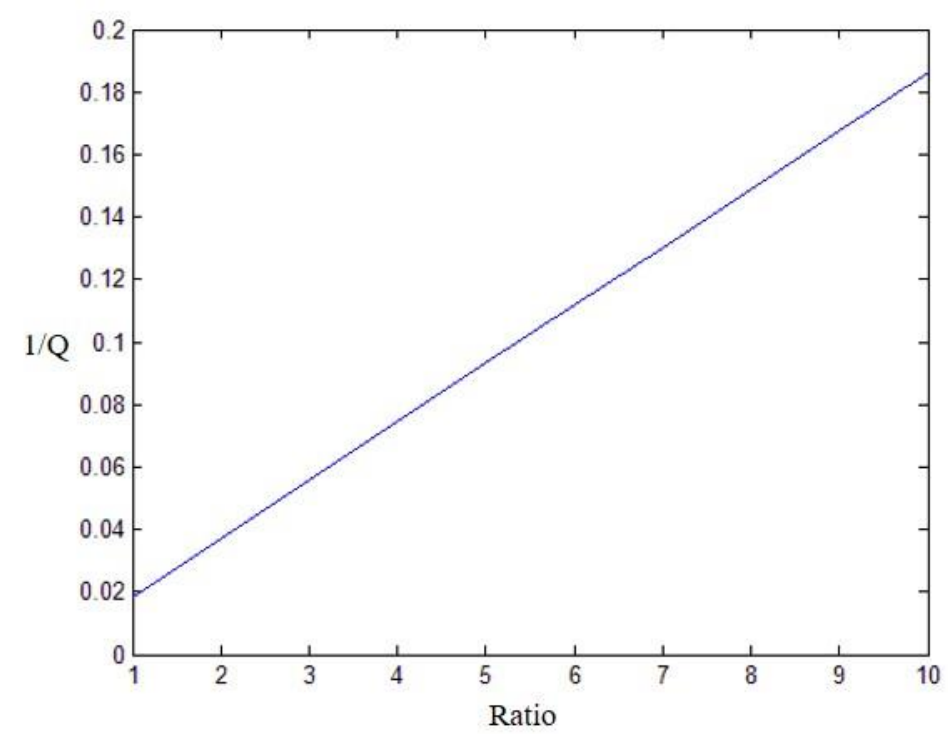

Fig. 6 Thermoelastic dissipation varies with the ratio of the height of the resonant ring and support structure

Thermoelastic dissipation varies with the ratio of the thickness of the resonant ring and support structure is showed in Fig. 7. It increases with the increase of the ratio of the height of the resonant ring and support structure, so when the thickness of the resonant ring is set a fixed value, the thicker the resonant ring, the thicker the thermoelastic dissipation. 


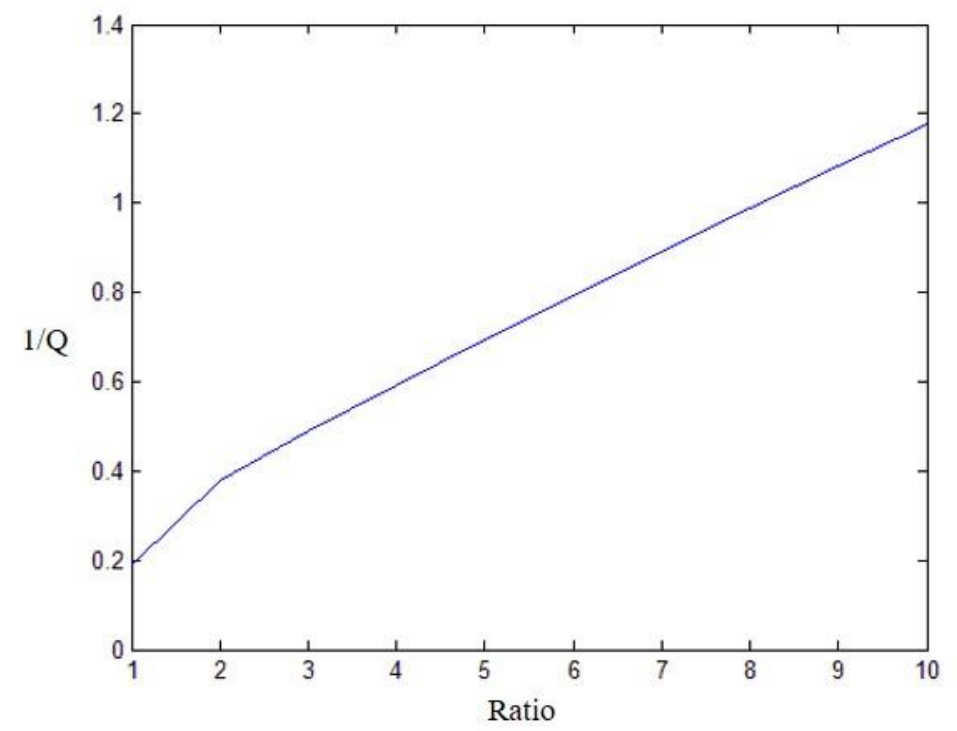

Fig. 7 Thermoelastic dissipation varies with the ratio of the thickness of the resonant ring and support

\subsection{Internal friction loss}

structure

In the process of vibration, the resonator needs to overcome viscous work, which produce the internal friction loss.

According to the definition [16], the internal friction loss of the resonator equals to

$$
\frac{1}{Q_{f r i}}=\frac{U_{f r i}}{2 \pi S}
$$

$U_{f r i}$ is the internal friction loss in a cycle and it contains the parts of radial deformation of the side wall $U_{s r}$, the axial deformation of the side wall $U_{s x}$ and the bottom surface $U_{s b}$.

$$
\begin{aligned}
& U_{s r}=\frac{1}{24} \frac{E \xi h_{s}^{3}}{R^{3}} \int_{0}^{L} \int_{0}^{2 \pi}\left(\frac{\partial^{2} v}{\partial \theta \partial t}-\frac{\partial^{3} w}{\partial^{2} \theta \partial t}\right) \cdot\left(\frac{\partial v}{\partial \theta}-\frac{\partial^{2} w}{\partial^{2} \theta}\right) d \theta d x \\
& U_{s x}=\frac{1}{24} E R \xi h_{s}^{3} \int_{0}^{L} \int_{0}^{2 \pi} \Delta k_{x} \cdot \frac{d \Delta k_{x}}{d t} d \theta d x \\
& U_{s b}=\frac{1}{24} E \xi h_{b}^{3} \int_{r_{0}}^{R} \int_{0}^{2 \pi} \Delta k_{b} \cdot \frac{d \Delta k_{b}}{d t} \cdot r d \theta d r
\end{aligned}
$$

$\zeta$ is the internal friction damping coefficient, and $\Delta k_{x}=\frac{W(\mathrm{x}))^{\prime} \cos 2 \theta \cos (\omega t)}{\left\{1+\left[W(\mathrm{x})^{\prime} \cos 2 \theta\right]^{2}\right\}^{3 / 2}}$, $\Delta k_{b}=\frac{\mathrm{U}_{b}(\mathrm{r}){ }^{\prime \prime} \cos 2 \theta \cos (\omega t)}{\left\{1+\left[\mathrm{U}_{b}(\mathrm{r})^{\prime} \cos 2 \theta\right]^{2}\right\}^{3 / 2}}$.

Internal friction loss, as showed in Fig. 8, its order of magnitude is about $10^{-5}$ to $10^{-4}$ and it increases with the increase of the outer radius of the resonant ring. 


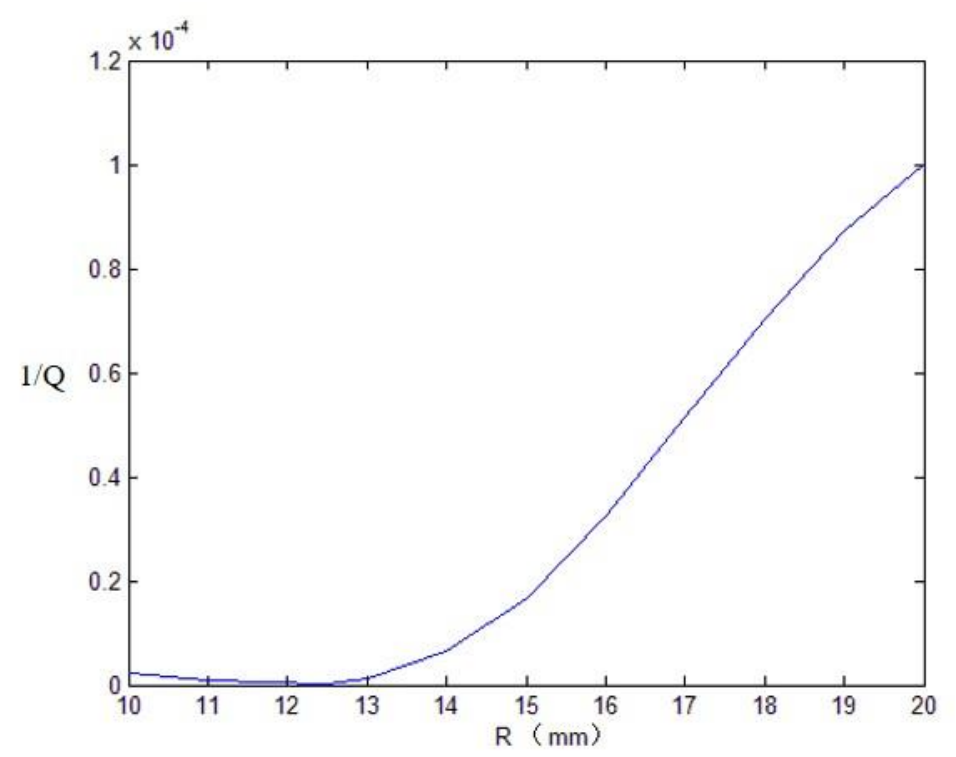

Fig. 8 Internal friction loss varies with the outer radius of the resonant ring

\subsection{General analysis}

When the outer radius of the resonant ring increases from $10 \mathrm{~mm}$ to $12.5 \mathrm{~mm}$, the internal friction loss decreases slowly. But when the radius is bigger than $13 \mathrm{~mm}$, the internal friction loss increases drastically. Since the order of magnitude of surface defect loss is about $10^{-5}$, the relative change is rather small when the outer radius varies. In addition, the thermoelastic loss is mainly affected by the material, so when the processing material is fixed, changing the internal friction loss can make a greater impact on the $\mathrm{Q}$ factor of the resonator. Therefore, $12.5 \mathrm{~mm}$, which corresponds with the smallest internal friction loss, is set in the radius of the resonant ring production. Other processing sizes are also set afterwards.

\section{Experiments}

\subsection{Annealing}

The common annealing process mainly consists of three processes, heating, heat preservation and cooling [17]. Annealing can eliminate the inherent stress of the material and the residual stress generated during the machining process, which reduce the thermoelastic dissipation and the internal friction loss.

In annealing experiments, the cylindrical shell fused silica resonator is placed in a SK-G08163 vacuum tube type furnace produced by Tianjin Central Electric Furnace Co., Ltd. The stress release temperature of the material is $1050{ }^{\circ} \mathrm{C}$, so the heating rate is set $200{ }^{\circ} \mathrm{C}$, the heat-preservation temperature is $1050{ }^{\circ} \mathrm{C}$ for 12 hours and the cooling rate is set $45{ }^{\circ} \mathrm{C} / \mathrm{h}$.

Measurement results before annealing are showed in table 2 . The resonant frequency and the Q factor are measured 5 times to get the average value. The resonant frequency is 3465.4 and the Q factor is 4375.7 .

Table. $2 \mathrm{Q}$ factor before annealing

\begin{tabular}{|c|c|c|}
\hline Groups & Resonant Frequency $[\mathrm{Hz}]$ & $Q$ factor \\
\hline 1 & 3465.4 & 4338.6 \\
\hline 2 & 3465.4 & 4386.5 \\
\hline 3 & 3465.4 & 4382.6 \\
\hline 4 & 3465.4 & 4384.5 \\
\hline 5 & 3465.4 & 4386.5 \\
\hline Average & 3465.4 & 4375.7 \\
\hline Standard deviation & 0 & 20.8 \\
\hline
\end{tabular}


Measurement results after annealing is showed in table 3. The resonant frequency raises to 3466.2 and the $\mathrm{Q}$ factor raises to 6489.6 , improves $48.3 \%$.

Table. 3 Q factor after annealing

\begin{tabular}{|c|c|c|}
\hline Groups & Resonant Frequency $[\mathrm{Hz}]$ & $Q$ factor \\
\hline 1 & 3466.2 & 6562.4 \\
\hline 2 & 3466.2 & 6389.9 \\
\hline 3 & 3466.2 & 6425.9 \\
\hline 4 & 3466.2 & 6557.3 \\
\hline 5 & 3466.2 & 6512.5 \\
\hline Average & 3466.2 & 6489.6 \\
\hline Standard deviation & 0 & 78.1 \\
\hline
\end{tabular}

Therefore, annealing can improves the $\mathrm{Q}$ factor of resonators obviously and this is realized by reducing the residual stress in the quartz resonator, which brings down the thermoelastic dissipation and the internal friction loss.

\subsection{Grinding the bottom}

Changing the material and size of resonators can affect the $\mathrm{Q}$ factor evidently. A resonator is tested before and after grinding the bottom. Each time the bottom thickness decreases $1 \mathrm{~mm}$ and its measurement results are showed in table 4, table 5, table 6 and table 7 .

Table. $4 \mathrm{Q}$ factor before grinding

\begin{tabular}{|c|c|c|}
\hline Groups & Resonant Frequency[Hz] & $Q$ factor \\
\hline 1 & 3506.8 & 4338.6 \\
\hline 2 & 3505.7 & 4141.7 \\
\hline 3 & 3506.7 & 4134.9 \\
\hline 4 & 3506.7 & 3975.7 \\
\hline 5 & 3506.7 & 4051.8 \\
\hline Average & 3506.5 & 4128.5 \\
\hline Standard deviation & 0.5 & 135.6 \\
\hline
\end{tabular}

\begin{tabular}{|c|c|c|}
\hline \multicolumn{3}{|c|}{ Table. 5 Q factor after first grinding } \\
\hline Groups & Resonant Frequency $[\mathrm{Hz}]$ & $Q$ factor \\
\hline 1 & 3490.9 & 5386.4 \\
\hline 2 & 3490.9 & 5460.3 \\
\hline 3 & 3491.1 & 5520.1 \\
\hline 4 & 3491.1 & 5575.1 \\
\hline 5 & 3491.1 & 5378.4 \\
\hline Average & 3491.1 & 5464.1 \\
\hline Standard deviation & 0.10 & 84.9 \\
\hline
\end{tabular}

Table. 6 Q factor after second grinding

\begin{tabular}{|c|c|c|}
\hline Groups & Resonant Frequency $[\mathrm{Hz}]$ & $Q$ factor \\
\hline 1 & 3483.8 & 5573.9 \\
\hline 2 & 3483.8 & 5586.9 \\
\hline 3 & 3483.7 & 5624.2 \\
\hline 4 & 3483.7 & 5593.9 \\
\hline 5 & 3483.7 & 5552.1 \\
\hline Average & 3483.7 & 5586.2 \\
\hline Standard deviation & 0.05 & 26.5 \\
\hline
\end{tabular}

\begin{tabular}{|c|c|c|}
\hline \multicolumn{3}{|c|}{ Table. 7 Q factor after third grinding } \\
\hline Groups & Resonant Frequency $[\mathrm{Hz}]$ & $Q$ factor \\
\hline 1 & 3459.2 & 3613.6 \\
\hline 2 & 3459.2 & 3792.1 \\
\hline 3 & 3459.3 & 3652.8 \\
\hline
\end{tabular}




\begin{tabular}{|c|c|c|}
\hline 4 & 3459.3 & 3786.9 \\
\hline 5 & 3459.4 & 3967.8 \\
\hline Average & 3459.3 & 3726.6 \\
\hline Standard deviation & 0.08 & 139.5 \\
\hline
\end{tabular}

After the first grinding, the $\mathrm{Q}$ factor increases from 4128.5 to 5464,5 , improves $32.4 \%$. After the second grinding, it reaches 5586.2, improves $2.3 \%$. But when the third grinding is finished, the Q factor drops to 3726.6 , deteriorates $33.2 \%$. Therefore, changing the size of resonators can affect the Q factor evidently and there is an optimum thickness which can get the biggest $\mathrm{Q}$ factor. This is realized by reducing the anchor loss when grinding the bottom. There is a critical thickness, when the bottom thickness is less than it, other damping loss caused from vibration mass reduction may surpass the reducing anchor loss and deteriorates the $\mathrm{Q}$ factor.

\subsection{Chemical polishing}

Chemical polishing, in other words, chemical etching, is a kind of method to remove the damaged layer on the surface of quartz by chemical solution, which can effectively reduce the surface defect loss of the material.

In chemical polishing experiments, the resonator is placed in a chemical etching solution mixed by $\mathrm{NH}_{4} \mathrm{~F}$ solution and $\mathrm{HF}$ solution for 10 minutes. Measurement results after chemical polishing are showed in table 8.

Table. $8 \mathrm{Q}$ factor after chemical polishing

\begin{tabular}{|c|c|c|}
\hline Groups & Resonant Frequency $[\mathrm{Hz}]$ & $Q$ factor \\
\hline 1 & 3146.9 & 8477.4 \\
\hline 2 & 3146.9 & 8523.7 \\
\hline 3 & 3146.9 & 8543.7 \\
\hline 4 & 3147.0 & 8609.3 \\
\hline 5 & 3147.0 & 8740.1 \\
\hline Average & 3146.9 & 8578.8 \\
\hline Standard deviation & 0.05 & 91.1 \\
\hline
\end{tabular}

After chemical polishing, the Q factor increases from 3726.6 to 8578.8 , improves $130.2 \%$. Therefore, chemical polishing can affect the $\mathrm{Q}$ factor evidently and this is realized by reducing the surface defect loss of the resonator.

\subsection{Vacuum experiments}

Air flow will greatly affect the vibration state of the resonator, which can lead to the air damping, so that the $\mathrm{Q}$ factor can be greatly reduced when the resonator is placed in a vacuum environment. Using the vacuum package, the vacuum degree reaches $10^{-2}$. Measurement results after vacuum pumping are showed in table 9.

Table. $9 \mathrm{Q}$ factor after vacuum pumping

\begin{tabular}{|c|c|c|}
\hline Groups & Resonant Frequency $[\mathrm{Hz}]$ & $Q$ factor \\
\hline 1 & 3157.8 & 467910.4 \\
\hline 2 & 3157.7 & 487278.9 \\
\hline 3 & 3157.7 & 474957.2 \\
\hline 4 & 3157.7 & 456887.3 \\
\hline 5 & 3157.8 & 420834.7 \\
\hline Average & 3157.7 & 461573.7 \\
\hline Standard deviation & 0.05 & 22634.7 \\
\hline
\end{tabular}

After vacuum pumping, the $Q$ factor increases from 8578.8 to 461573.7 , improves about 53 times. Therefore, vacuum pumping can greatly improve the $\mathrm{Q}$ factor of the resonator and this is realized by reducing the air damping.

After bottom grinding, chemical polishing and vacuum pumping, the $\mathrm{Q}$ factor of the resonator has been optimized evidently, increasing from 4128.5 to 461573.7 , improves about 110 times. The next step is to develop an annealing solution for the material and size, the Q factor is expected to have 
futher increase. In addition, the bottom grinding scheme can be optimized because the third grinding has decreased the $\mathrm{Q}$ factor, otherwise the $\mathrm{Q}$ factor can be even higher after vacuum pumping.

\section{Conclusions}

This paper focuses on the quality factor of the cylindrical shell fused silica resonator. Firstly, a new resonator model is proposed, containing the bottom vibration of the resonator based on the beam model. Then, the influence of air damping, surface defect loss, thermoelastic dissipation and internal friction loss on the quality factor are studied through simulations, the processing sizes are also set according to the simulation results. The $\mathrm{Q}$ factor is further explored by means of changing different damping loss of the resonator, after bottom grinding, chemical polishing and vacuum pumping, the $\mathrm{Q}$ factor of the resonator has been optimized evidently, increasing from 4128.5 to 461573.7, improves about 110 times.

\section{References}

[1] Chikovani V V, Yatsenko Y A, Barabashov A S, et al. Improved accuracy metallic resonator CVG,J. Aerospace and Electronic Systems Magazine, IEEE (2009) 24(5): 40-43.

[2] Chikovani V V, Yatzenko Y A, Kovalenko V A. Coriolis force gyroscope with high sensitivity: U.S.Patent7281425.( 2007)

[3] Rozelle D M. The hemispherical resonator gyro: From wineglass to the planets, C.Proc. 19th AAS/AIAA Space Flight Mechanics Meeting. (2009)1157-1178.

[4] Xi X, Wu X, Zhang Y, et al. A study on Q factor of the trimmed resonator for vibratory cupped gyroscopes, J. Sensors \& Actuators A Physical, (2014)218(10) 23-32.

[5] Watson W S. Vibratory gyro skewed pick-off and driver geometry, C. Position Location and Navigation Symposium (PLANS), IEEE/ION. IEEE(2010)171-179.

[6] Chikovani V V, Okon I M, Barabashov A S, et al. A set of high accuracy low cost metallic resonator CVG, C. Position, Location and Navigation Symposium, IEEE/ION. IEEE(2008)238-243.

[7] T. L. Floyd. Electric Circuits Fundamentals[M]. Prentice hall, 2009.

[8] R. Abdolvand, H. Johari, G. K. Ho, F. Ayazi. Quality factor in trench-refilled polysilicon beam resonators[J]. Journal of Microelectromechanical Systems, 2006, 15(3), 1-6.

[9] Yao P, Dongya W, Yanyan W, et al. Monolithic cylindrical fused silica resonators with high Q factors, Sensors(2016).

[10] Silva De, Clarence W, ed. Vibration damping, control, and design. CRC Press, 2007.

[11] N. Liu, Z. Su, Q. Li, M. Y. Fu, H. Liu, J. F. Fan. Characterization of the Bell-Shaped Vibratory Angular Rate Gyro[J]. Sensors, 2013, 13: 10123-10151.

[12] T. Uchiyama, T. Tomaru, M. Tobar, D. Tatsumi, S. Miyoki, M. Ohashi. Mechanical quality factor of a cryogenic sapphire test mass for gravitational wave detectors[J]. Physics Letters A, 1999, 261: 5-11.

[13] Choi J, Cho M, Rhim J. Efficient prediction of the quality factors of micromechanical resonators[J]. Journal of Sound \& Vibration, 2010, 329(1):84-95.

[14] Choi S Y, Na Y H, Kim J H. Thermoelastic Damping of Inextensional Hemispherical Shell[J]. World Academy of Science Engineering \& Technology, 2009, (56).

[15] Zener C. Elasticity and anelasticity of metals[M]. Chicago: Univ. Chicago Press, 1948: 175-180. 
[16] Xiang Xi, Xuezhong Wu, Yongmeng Zhang, Zhou Xin, Xiaomei Wu, Yulie Wu. A Study on Q Factor of The Trimmed Resonator for Vibratory Cupped Gyroscopes[J]. Sensors and Actuators A: Physical, 2014, 218: 23-32.

[17] Ahamed M J, Senkal D, Shkel A M. Effect of annealing on mechanical quality factor of fused quartz hemispherical resonator[C]// Inertial Sensors and Systems (ISISS), 2014 International Symposium on. IEEE, 2014:1-4. 